\title{
Reincidência da gravidez em adolescentes de Teresina, PI, Brasil
}

\author{
Relapse into preganancy in adolescents from Teresina, PI, Brazil \\ Reincidencia del embarazo en adolescentes de Teresina, PI, Brasil
}

\author{
Inez Sampaio Nery', Rita de Cássia Magalhães Mendonça', \\ Ivanilda Sepúlveda Gomes", Ana Catharina Nunes Fernandes ${ }^{\text {III }}$, Delviane Costa de Oliveira" ${ }^{\text {IV }}$ \\ 'Universidade Federal do Piauí. Departamento de Enfermagem. Teresina, PI \\ "Fundação Municipal de Saúde. Teresina, PI

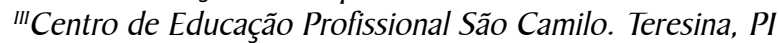 \\ "vaculdade Santo Agostinho. Teresina, PI
}

Submissão: $21 / 09 / 2009$

Aprovação: 07/1 1/2010

\section{RESUMO}

A gravidez na adolescência é um problema de saúde pública, tornando-se mais grave em virtude da reincidência. De 1,1 milhões de parturientes adolescentes, $25 \%$ tem um filho. Objetivou-se analisar os fatores sócio-econômico-cultural e obstétrico da reincidência de gravidez na adolescência em Teresina, PI, Brasil por meio de um estudo interrelacional retrospectivo. A amostra incluiu 464 jovens Que finalizaram uma gestação no primeiro Quadrimestre de 2006. Destas, 47,6\% encontrava-se em união consensual; 69,6\% fora da escola; $86,9 \%$ com escolaridade inadequada para a idade; $72,2 \%$ sem trabalho remunerado. Este perfil evidencia a escassez de políticas públicas Que contemplem o adolescente. A elaboração e efetivação dessas políticas deveriam incluir família, escola e comunidade para redução dos indicadores de gravidez e doenças sexualmente transmissíveis.

Descritores: Gravidez na adolescência; Saúde sexual e reprodutiva; Enfermagem.

\section{ABSTRACT}

Pregnancy in adolescence is a public health problem, and relapse rates make it even more serious. Out of 1,1 million parturient adolescents, $25 \%$ have a child already. We aimed to analyze the social economic cultural obstetric factors of relapse into pregnancy in adolescence in Teresina, PI, Brazil through interelational retrospective study. The sample contains 464 young who finished a gestation during the first four months in 2006. Out of those, $47.6 \%$ were in consensual union; $69.6 \%$ out of school; $86.9 \%$ with inappropriate schooling for the age; $72.2 \%$ with unpaid job. This profile evidences the lack of public policies that consider the adolescent. The elaboration and development of those policies should include family, school and the community in order to decrease pregnancy and sexually-transmited diseases rates.

Key words: Pregnancy in adolescence; Sexual and reproductive health; Nursing.

\section{RESUMEN}

El embarazo en la adolescencia es un problema de salud pública, tornándose más grave en virtud de la reincidencia. De 1,1 millones de parturientes adolescentes, 25\% ya tienen un hijo. Objetivamos analizar los factores socios económicos culturales y obstétricos de la reincidencia del embarazo en la adolescencia en Teresina, PI, Brazil por medio de un estudio interrelacional retrospectivo. La muestra incluye 464 jóvenes Que finalizaron una gestación en el primer cuadrimestre de 2006. De estas, 47,6\% estaban en unión consensual; $69.6 \%$ fuera de la escuela; $86,9 \%$ con escolaridad inadecuada para la edad; $72,2 \%$ sin trabajo remunerado. Este perfil evidencia la escasez de políticas públicas que contemplen el adolescente. La elaboración y efectuación de esas políticas deberían incluir la familia, la escuela y la comunidad para reducción de los indicadores de embarazo y enfermedades sexualmente transmisibles.

Descriptores: Embarazo en la adolescencia; Salud sexual y reproductiva; Enfermeria.

AUTOR Inez Sampaio Nery. Campus Universitário Ministro Petrônio Portella. Ininga. CEP 64049-550. Teresina, PI.

CORRESPONDENTE_E-mail ineznery.ufpi@gmail.com 


\section{INTRODUÇÃO}

De acordo com os critérios da Organização Mundial de Saúde (OMS), a adolescência é o período compreendido entre 10 a 19 anos $^{(1)}$. Este período marca o início da vida reprodutiva e caracterizase por mudanças fisiológicas corporais e psicológicas na adolescência. Tais transformações e adaptações devem transcorrer de forma saudável, a fim de Que não tragam malefícios ao adolescente, Quanto a sua saúde física, mental, social e espiritual ${ }^{(2)}$.

Atualmente existe no mundo mais de um bilhão de pessoas com idade entre 10 e 19 anos, o Que representa Quase 20\% da população mundial. No Brasil, esse número compreende 35 milhões de adolescentes de ambos os sexos entre 10 e 19 anos. No Estado do Piaú há 749.021 adolescentes de 10 a 19 anos, de ambos os sexos ${ }^{(3)}$.

A Classificação da adolescência por faixa etária é uma estratégia para definição de políticas e alocação de recursos e muitas vezes apresentam-se de forma divergente entre os estudiosos. A delimitação cronológica da adolescência é influenciada por fatores socioculturais, familiares e pessoais ${ }^{(4)}$.

Especialistas em adolescência alertam que de I, I milhões de adolescentes parturientes de 15 a 19 anos no Brasil, 25\% já tem um filho. $\mathrm{O}$ fato mais preocupante é Que grande parte das mesmas afirma Que a sucessiva gravidez não foi planejada. Um dos fatores Que tem sido apontado como importante na recorrência da gravidez entre os jovens é a negligência Quanto à contracepção, considerando-se Que adolescentes com vida sexual ativa estão expostos a uma nova gravidez dentro de um ano se não for utilizado nenhum método contraceptivo, com chance de nove para cada dez adolescentes. Outro fator Que contribui para repetidas gestações é a antecipação da primeira relação sexual, acontecendo hoje, em média, aos 13 anos de idade ou menos ${ }^{(5)}$.

As desigualdades entre o masculino e o feminino surgem a partir de dinâmicas culturais Que estabelecem socialmente uma condição de inferioridade para a mulher, Que muitas vezes reproduz os papéis sexuais de acordo com o meio sócio-cultural em Que estão inseridas, sem posicionamento crítico em relação aos padrões de conduta pré-estabelecidos pelos pais e sociedade. Essa Questão de gênero influencia na restrição de práticas sexuais para a menina, enQuanto para o menino há estímulo à iniciação sexual precoce ${ }^{(2)}$. A falta de um projeto de vida para as jovens de classes mais pobres, fora do casamento demonstra Que há poucas perspectivas para essas jovens, o que resulta no abandono dos estudos. Algumas destas garotas até reconhecem que a falta de perspectivas de vida, aliada aos conflitos familiares, as levam à busca de maior autonomia, Que muitas vezes se processa em uma gravidez não planejada ou na sua recorrência ${ }^{(6)}$.

Outro fator Que tem contribuído para o aumento da ocorrência e recorrência de gravidez na adolescência é a modificação dos padrões de sexualidade, visto Que a iniciação sexual ocorre cada vez mais precocemente, em especial nos países em desenvolvimento. No Brasil, estima-se Que anualmente há um milhão de partos envolvendo adolescentes, o Que correspondeu a 25,79\% do total de nascidos vivos em 1996. Em 1995, o Sistema Único de Saúde (SUS) gastou 153 milhões de reais, o que equivale a $27 \%$ de todos os partos atendidos no sistema, com gestações em adolescentes ${ }^{(7)}$.

Vale destacar Que em estudo sobre reincidência de gravidez na adolescência, realizado em 2000 na cidade de São Paulo, percebeu- se Que a mesma não decorre da falta de conhecimentos dessas jovens sobre os métodos contraceptivos. Neste levantamento, constatou-se Que $98 \%$ das adolescentes têm conhecimentos sobre a utilização da pílula e $99,4 \%$ sobre o condom (preservativo masculino). O estudo enfatiza ainda Que os programas de combate a AIDS sejam os principais disseminadores de informações sobre a utilização do condom. Um percentual significativo de $10,4 \%$ alegou a não utilização de métodos contraceptivos por acreditarem Que seriam incapazes de engravidar. $\mathrm{E}$ a taxa de reincidência entre as adolescentes do estudo foi de $21,2 \%{ }^{(8)}$.

Ao investigar as possíveis causas reais da gravidez na adolescência e sua recorrência, de acordo com a análise dos fatores predisponentes, presume-se Que é possível formular estratégias eficazes para reduzir esta problemática Que acomete a população brasileira, além de abrir horizontes para estudos posteriores Que busquem o mesmo ideal. Neste contexto, esse estudo teve como objetivos identificar os fatores de reincidência da gravidez na adolescência e analisar os fatores sócio-econômicos, culturais, obstétricos e os motivos apontados pela adolescente para a reincidência de gravidez até dois anos após o término de uma gestação.

\section{MÉTODOS}

Tratou-se de estudo interrelacional retrospectivo, realizado no município de Teresina, PI. Optou-se pelo estudo retrospectivo tendo em vista Que assim se poderia estudar o período de dois anos após a finalização de gravidez ocorrida em 2006. A macro-região de Teresina-PI, capital do Estado está dividida em três regiões de saúde, tais como: Centro-Norte, Sul, Leste-Sudeste. A população inicial do estudo foi formada por todas as adolescentes que finalizaram uma gravidez nos primeiros Quatro meses de 2006, Quando tinham idade de 15 a 19 anos, em seis hospitais - maternidades de Teresina. Destas, foram incluídas apenas as residentes em área urbana. Finalizado o levantamento, obteve-se uma amostra de 464 jovens. Segundo dados da Súmula Informativa da FMS ${ }^{(9)}$, no ano de 2006 nasceram 2.946 crianças vivas de mães adolescentes e residentes no município, o Que correspondeu a 20,29\% de todos os partos de mães residentes.

A população de adolescentes do sexo feminino de 15 a 19 anos no Piauí no ano de 2005 foi de 184.439 pessoas. Dados do Sistema de Informação de Nascidos Vivos - SINASC mostram Que nos municípios em Que se farão coleta de dados nasceram 1.54l crianças de mães com idade de 15 a 19 anos, em 2005, último ano com dados disponíveis no sítio eletrônico do Departamento de Informática do SUS - Datasus. Tendo em vista Que o SISPRÉNATAL não tem cobertura satisfatória no Piauí, e Que não é possível localizar-se estes casos para o município de Teresina acessando-os pelo nível de Atenção Básica, optou-se pela utilização dos dados gerados nas maternidades. A inclusão da amostra selecionada de adolescentes Que há dois anos pertenciam a faixa etária de 15 a 19 anos se deu para facilitar a comparação com outros estudos, haja vista Que a ocorrência e recorrência da gravidez nesta população etária são significativamente superiores aos casos ocorridos na faixa etária de 10 a 14 anos. Neste sentido, vale ressaltar Que em 2005, o nascimento de crianças de mães nesta faixa etária, foi 29 vezes menor Que o número de nascimento de mães na faixa etária de 15 
a $19 \operatorname{anos}^{(10)}$.

As adolescentes foram identificadas a partir de dados registrados nas maternidades. Os casos não localizados foram incorporados a uma listagem e encaminhados às enfermeiras da Unidade Básica de Saúde (UBS) correspondente à área de cobertura onde residia à adolescente, para Que fosse verificada sua provável localização. Eram feitas até cinco tentativas para localizar os casos. Somente na ocasião em Que não se obtinha sucesso na busca, o caso era considerado perdido.

Para a coleta de dados, utilizou-se um formulário semiestruturado aplicado por meio da técnica de entrevista, visando levantar dados pertinentes aos objetivos da pesquisa. $\mathrm{O}$ instrumento foi constituído em três partes: a primeira abrange variáveis sociodemográficas; a segunda contém os antecedentes reprodutivos; e a terceira se refere aos motivos relacionados à reincidência de gravidez e conhecimentos acerca da contracepção.

A coleta de dados foi realizada nos meses de maio a setembro de 2008 por 16 acadêmicas do Curso de Enfermagem da Universidade Federal do Piauí, dentre elas oito bolsistas e oito voluntárias Que foram treinadas para a tarefa, sob supervisão de enfermeiras-pesquisadoras Que colaboraram com o estudo, e supervisão geral das pesquisadoras responsáveis na coleta de dados.

Previamente à coleta de dados, procedeu-se contato com os gestores estaduais e municipais das instituições de saúde para receber documento de autorização para execução da coleta de dados para a pesquisa.

A presente pesquisa é um recorte de um projeto desenvolvido junto ao Conselho Nacional de Desenvolvimento Científico e Tecnológico - CNPQ. Esta pesquisa também obedeceu aos princípios ético-legais, no momento em Que se apresentava aos sujeitos o Termo de Consentimento Livre e Esclarecido com base na Resolução n 1 96/96 do Conselho Nacional de Saúde - MS, referente à pesquisa envolvendo seres humanos. A pesquisa foi aprovada pelo Comitê de Ética e Pesquisa da UFPI, conforme o Parecer No 56/08, CONEP e Instituições Municipais de Saúde.

A validação do banco de dados foi realizada através do aplicativo Epi Info, versão 6.04d (U.S. Center for Disease Control and Prevention, Atlanta, GA). Os dados foram submetidos ao tratamento estatístico, através da codificação e tabulação, utilizando-se o Programa SPSS para Windows Versão 17.0, o Qual permite a tabulação das informações obtidas e análise por meio de estatística descritiva bivariada para este estudo. De posse das variáveis dispostas em tabelas, iniciou-se o trabalho de análise e discussão dos resultados a luz da literatura local, nacional e internacional acerca do tema.

\section{RESULTADOS}

Os resultados compreendem a caracterização dos sujeitos e os fatores sócio-econômico-cultural e obstétrico da reincidência de gravidez na adolescência em TeresinaPI contidos nas Tabelas I a 5.

O grupo participante tinha idade de 17 a 22 anos, com média de 20,1 anos, desvio padrão de 1,3 e idade mediana de 20 anos (não mostrados). Na Tabela I, com dados sobre características demográficas, é mostrado Que cerca de 70\% das jovens já não eram adolescentes e tinham de 20 a 22 anos de idade. Sessenta e cinco e meio do grupo estava em união consensual ou eram casadas, sendo Que de todo o grupo $63,6 \%$ relatou morar com o companheiro e cerca de $1 / 4$ não mantinham os laços conjugais com o ex-companheiro. A religião católica foi predominante com $81,2 \%$ delas informando terem esta religião. Quase 70\% das jovens não estudavam, sendo Que $94,4 \%$ do grupo geral haviam abandonado os estudos em algum momento da vida, mas $58,7 \%$ destas jovens jamais retornaram a estudar. Os níveis de escolaridade predominantes foram o ensino fundamental incompleto com $34,7 \%$ das jovens tendo informado este nível e o ensino médio incompleto com 32, I\%. O nível de escolaridade para Quase $87,0 \%$ das entrevistadas não era adeQuado à idade

Na Tabela 2 mostram-se características econômicas das entre-

Tabela 1. Distribuição de adolescentes e jovens com antecedentes gestacionais residentes em Teresina-PI, segundo características demográficas. 2008.

\begin{tabular}{|c|c|c|}
\hline Variáveis & $\mathrm{n}$ & $\%$ \\
\hline \multicolumn{3}{|l|}{ Faixa etária (anos) } \\
\hline 17 a 19 & 140 & 30,2 \\
\hline 20 a 22 & 324 & 69,8 \\
\hline \multicolumn{3}{|l|}{ Situação conjugal } \\
\hline União estável & 221 & 47,6 \\
\hline Solteira & 152 & 32,8 \\
\hline Casada & 83 & 17,9 \\
\hline Divorciada & 6 & 1,3 \\
\hline Viúva & 2 & 0,4 \\
\hline \multicolumn{3}{|l|}{ Mora com o companheiro } \\
\hline Sim & 295 & 63,6 \\
\hline Não e não mantém os laços conjugais & 108 & 23,3 \\
\hline Não, mas mantém laços conjugais & 61 & 13,1 \\
\hline \multicolumn{3}{|l|}{ Religião } \\
\hline Católica & 377 & 81,2 \\
\hline Evangélica & 51 & 11,0 \\
\hline Outra & 4 & 0,9 \\
\hline Sem religião & 32 & 6,9 \\
\hline \multicolumn{3}{|l|}{ Estuda } \\
\hline Sim & 141 & 30,4 \\
\hline Não & 323 & 69,6 \\
\hline \multicolumn{3}{|l|}{ Parou de estudar em algum momento } \\
\hline Sim & 438 & 94,4 \\
\hline Não & 26 & 5,6 \\
\hline \multicolumn{3}{|l|}{ Voltou a estudar após parar } \\
\hline $\operatorname{Sim}$ & 181 & 41,3 \\
\hline Não & 257 & 58,7 \\
\hline \multicolumn{3}{|l|}{ Nível de escolaridade } \\
\hline Analfabeta funcional ( $<4$ anos de estudo) & 6 & 1,3 \\
\hline Ensino fund incompleto (? $4 \mathrm{a}$ ? 7 anos de est) & 161 & 34,7 \\
\hline Ensino fundamental & 55 & 11,9 \\
\hline Ensino médio incompleto & 149 & 32,1 \\
\hline Ensino médio & 82 & 17,7 \\
\hline Ensino superior (incompleto/completo) & 11 & 2,4 \\
\hline \multicolumn{3}{|l|}{ AdeQuação idade-série } \\
\hline $\mathrm{Sim}$ & 61 & 13,1 \\
\hline Não & 403 & 86,9 \\
\hline
\end{tabular}


Tabela 2. Distribuição de adolescentes e jovens com antecedentes gestacionais residentes em Teresina-PI, segundo características econômicas. 2008.

\begin{tabular}{|c|c|c|}
\hline Variáveis & $\mathrm{n}$ & $\%$ \\
\hline \multicolumn{3}{|l|}{ Trabalho remunerado } \\
\hline Sim & 129 & 27,8 \\
\hline Não & 335 & 72,2 \\
\hline \multicolumn{3}{|l|}{ Renda Familiar } \\
\hline Até $1 / 2$ salário mínimo (SM) (até $\mathrm{R} \$ 206,00$ ) & 60 & 12,9 \\
\hline Até I SM (de 206,01 a R\$ 412,00$)$ & 172 & 37,1 \\
\hline Mais de I a 2 SM (de $R \$ 412,01$ a $R \$ 824,00$ ) & 135 & 29,1 \\
\hline Mais de 2 a 3 SM (de $R \$ 824,01$ a $1.236,00$ ) & 48 & 10,3 \\
\hline Mais de 3 SM (R\$ 1.236,01 ou mais) & 49 & 10,6 \\
\hline \multicolumn{3}{|l|}{ Dependência financeira } \\
\hline Sim, parcialmente & 101 & 21,8 \\
\hline Sim, totalmente & 300 & 64,7 \\
\hline Não & 63 & 13,6 \\
\hline \multicolumn{3}{|l|}{ De Quem depende financeiramente } \\
\hline Companheiro & 202 & 50,4 \\
\hline Pais dela & 111 & 27,7 \\
\hline Pais dele (companheiro) & 5 & 1,2 \\
\hline Outra(s) pessoa(s) & 83 & 20,7 \\
\hline
\end{tabular}

Tabela 3. Distribuição de adolescentes e jovens com antecedentes gestacionais residentes em Teresina-PI, segundo características familiares. 2008.

\begin{tabular}{|c|c|c|}
\hline Variáveis & $\mathrm{n}$ & $\%$ \\
\hline \multicolumn{3}{|l|}{ Escolaridade da mãe } \\
\hline Não alfabetizada & 66 & 14,2 \\
\hline Analfabeta funcional $(<4$ anos de estudo) & 54 & 11,6 \\
\hline Ensino fund incompleto (? 4 a? 7 anos de estudo) & 178 & 38,4 \\
\hline Ensino fundamental & 36 & 7,8 \\
\hline Ensino médio incompleto & 35 & 7,5 \\
\hline Ensino médio & 48 & 10,3 \\
\hline Ensino superior (incompleto/completo) & 12 & 2,6 \\
\hline Não sabe & 35 & 7,5 \\
\hline \multicolumn{3}{|l|}{$\begin{array}{l}\text { Idade com Que a mãe da jovem engravidou pela primeira } \\
\text { vez (anos) }\end{array}$} \\
\hline 11 a 14 & 40 & 8,6 \\
\hline 15 a 19 & 245 & 52,8 \\
\hline 20 a 24 & 142 & 30,6 \\
\hline 25 e mais & 27 & 5,7 \\
\hline Não sabe & 10 & 2,2 \\
\hline \multicolumn{3}{|l|}{$\begin{array}{l}\text { Irmã ou irmão da jovem teve filho antes dos } 20 \\
\text { anos de idade }\end{array}$} \\
\hline Sim & 217 & 48,1 \\
\hline Não & 234 & 51,9 \\
\hline
\end{tabular}

vistadas, em Que se observa Que $72,2 \%$ não informaram trabalho remunerado. As jovens viviam em famílias Que predominantemente ganhavam no máximo um salário mínimo $(37,1 \%)$ e Que juntamente com aQuelas Que informaram até $1 / 2$ salário mínimo de renda familiar, correspondem a $50 \%$ da amostra. Cerca de $65,0 \%$ das entrevistadas referiu depender financeiramente de terceiros, sendo o companheiro apontado por $50,4 \%$ das jovens como o responsável pelo sustento delas.

Características familiares das entrevistadas são mostradas na Tabela 3. A mãe de 64,2 destas jovens no máximo tinha o ensino fundamental incompleto como nível de escolaridade e apenas 2,6\% chegaram a ingressar em um curso superior. Pouco mais de $61 \%$ das mães havia engravidado na adolescência e cerca de $48 \%$ das jovens tinham pelo menos um irmão ou irmã Que também tiveram filhos na adolescência.

Dados sobre a iniciação sexual da jovem são mostrados na Tabela 4, onde pode ser observado Que a menarca predominou nas idades de 12 e 13 anos para $50,6 \%$ do grupo estudado. A primeira relação sexual com penetração ocorreu para a maioria das jovens Quando elas tinham de nove a Quinze anos de idade $(53,7 \%)$, com destaque para a idade de 15 anos $(24,4 \%)$ Que foi a mediana para esta variável. A maioria das entrevistadas informou Que seus pais não a orientaram sobre sexo antes da primeira relação sexual $(57,5 \%)$, enQuanto para a maioria a escola é Que fez esta orientação $(72,2 \%)$.

Na Tabela 5, Que mostra características obstétricas das entrevistadas, nota-se Que Quase $50 \%$ das jovens informaram Que havia engravidado uma vez na vida; Que 6,3\% estavam grávidas no momento da entrevista e Que houve reincidência de gravidez para pouco mais Que 1/4 das participantes do estudo. Cerca de $64 \%$ das jovens referiram ter apenas um filho vivo; Que a primeira gestação ocorreu predominantemente Quando elas estavam na faixa etária de 15 a 19 anos, mas todas experimentaram a primeira gestação na adolescência. Para 1/3 das jovens o parceiro das gestações seguintes à primeira foi um homem diferente daquele da primeira e que $54,5 \%$ destes novos parceiros não tinham filhos de relacionamentos anteriores.

\section{DISCUSSÃO}

O perfil detectado por este estudo para as adolescentes entrevistadas é semelhante a perfis encontrados por outros estudos nacionais ${ }^{(1-13)}$. Considerando que o principal critério de inclusão do estudo foi Que as jovens tivessem tido uma resolução de gravidez no primeiro Quadrimestre de 2006, Quando elas ainda eram adolescentes com idade de 15 a 19 anos, grupo significativo destas jovens já não eram adolescentes, mas permaneciam no grupo dos considerados jovens pela Organização Mundial de Saúde ${ }^{(1)}$.

Estimativa de 2006 era Que, no mundo, nos próximos dez anos, 100 milhões de adolescentes se casem antes de completarem 18 anos idade ${ }^{(14)}$. Países com menor renda per capita como Bangladesh e Burkina Faso têm no mínimo $60 \%$ de mulheres adolescentes casadas. O Brasil, apesar de renda per capita o classificar como país de renda média alta pelo Banco Mundial e o Haiti ser um país de renda baixa ${ }^{(15)}$, em se tratando de casamento precoce ambos pertencem ao mesmo grupo para a Organização Mundial de Saúde fazem do mesmo grupo. O grupo de países em Que pelo menos $20 \%$ das adolescentes estão casadas antes do décimo oitavo aniversário ${ }^{(14)}$.

O casamento precoce pode ser algo desejável para as famílias em sociedades economicamente desfavoráveis, em Que a filha pode se tornar um peso financeiro. Em outras sociedades, como a brasileira, o casamento precoce pode ser a solução para jovens 
Tabela 4, Distribuição de adolescentes e jovens com antecedentes gestacionais residentes em Teresina-PI, segundo características da iniciação sexual. 2008.

\begin{tabular}{|c|c|c|}
\hline Variáveis & $\mathrm{n}$ & $\%$ \\
\hline \multicolumn{3}{|c|}{ Idade da menarca (anos) } \\
\hline 09 a 11 & 87 & 18,8 \\
\hline 12 e 13 & 235 & 50,6 \\
\hline 14 a 18 & 142 & 30,6 \\
\hline \multicolumn{3}{|c|}{$\begin{array}{l}\text { Idade da primeira relação sexual com penetração } \\
\text { (anos) }\end{array}$} \\
\hline 9 a 14 & 136 & 29,3 \\
\hline 15 & 113 & 24,4 \\
\hline 16 e 17 & 179 & 38,6 \\
\hline 18 e 19 & 36 & 7,8 \\
\hline \multicolumn{3}{|c|}{$\begin{array}{l}\text { Os pais a orientaram sobre sexo antes da primeira } \\
\text { relação sexual }\end{array}$} \\
\hline Sim & 197 & 42,5 \\
\hline Não & 267 & 57,5 \\
\hline \multicolumn{3}{|c|}{$\begin{array}{l}\text { Na escola foi orientada sobre sexo seguro antes da } \\
\text { primeira relação dela }\end{array}$} \\
\hline Sim & 335 & 72,2 \\
\hline Não & 129 & 27,8 \\
\hline
\end{tabular}

Tabela 5. Distribuição de adolescentes e jovens com antecedentes gestacionais residentes em Teresina-PI, segundo características obstétricas. 2008.

\begin{tabular}{|c|c|c|}
\hline Variável & $\mathrm{n}$ & $\%$ \\
\hline \multicolumn{3}{|c|}{ Quantas vezes engravidou } \\
\hline Uma & 228 & 49,1 \\
\hline Duas & 146 & 31,5 \\
\hline Três & 64 & 13,8 \\
\hline Mais de três & 26 & 5,6 \\
\hline \multicolumn{3}{|c|}{ Grávida no momento da entrevista } \\
\hline Sim & 29 & 6,3 \\
\hline Não & 435 & 93,7 \\
\hline \multicolumn{3}{|c|}{ Gravidez reincidente nos últimos dois anos } \\
\hline Sim & 120 & 25,9 \\
\hline Não & 344 & 74,1 \\
\hline \multicolumn{3}{|c|}{ Quantidade de filhos vivos } \\
\hline Nenhum & 17 & 3,6 \\
\hline Um & 296 & 63,8 \\
\hline Dois & 116 & 25,0 \\
\hline Três e mais & 35 & 7,6 \\
\hline \multicolumn{3}{|c|}{ Idade na primeira gestação (anos) } \\
\hline $12 \mathrm{a} 14$ & 53 & 11,5 \\
\hline 15 a 19 & 411 & 88,5 \\
\hline \multicolumn{3}{|c|}{$\begin{array}{l}\text { Se o parceiro de gestações seguintes era o mesmo } \\
\text { da primeira * }\end{array}$} \\
\hline $\operatorname{Sim}$ & 157 & 66,5 \\
\hline Não & 79 & 33,5 \\
\hline \multicolumn{3}{|c|}{$\begin{array}{l}\text { Parceiro de gestaçóes seguintes à primeira tinha filhos antes } \\
\text { dela engravidar dele * }\end{array}$} \\
\hline Sim & 34 & 43,0 \\
\hline Não & 43 & 54,5 \\
\hline Não sabe & 2 & 2,5 \\
\hline
\end{tabular}

O casamento precoce é ainda considerado um fator Que acelera o surgimento de uma gravidez, até devido ser, muitas vezes, o motivo do casamento. Dados do Demographic and Health Survey (DHS) ou Peseuisa sobre Demografia e Saúde de 1996 mostraram Que $1 / 4$ dos nascidos vivos de adolescentes nordestinas nasceram sete meses após o casamento ${ }^{(17)}$.

Sobre a elevada freeüência de adolescentes em união consensual nesta pesquisa piauiense, dados comparativos do DHS dos anos de 1986, 1991 e 1996 realizadas no Brasil mostraram Que entre adolescentes nordestinas, a probabilidade de dissolução da união conjugal é significativamente maior nas uniões informais do Que nas formais ${ }^{(17)}$.

A escolaridade tem sido mostrada como a variável diferencial e resolutiva para a Questão social da gravidez na adolescência pela literatura especializada ${ }^{(17-18)}$. Estudos nacionais comprovam Que durante a gravidez na adolescência ou após o nascimento de um filho, significativa proporção de adolescentes abandona os estudos $^{(13,17-18)}$. Tem sido provado também Que entre adolescentes brasileiras Que são casadas e não têm filhos há maior probabilidade de abandonarem os estudos do Que aQuelas solteiras sem filhos ${ }^{(19)}$. A adolescência é uma fase em Que os indivíduos estão em formação, incluindo a escolar, e obtendo habilidades para maior chance de sucesso na vida adulta. $\mathrm{O}$ ingresso precoce no mercado de trabalho e assumir responsabilidades Que são mais adequadas para adultos, como cuidar de uma família, reduz as chances de continuar os estudos e de sucesso desta adolescente. Abandonar os estudos torna o futuro da jovem e de sua prole vulneráveis, restringe suas oportunidades de conseguir postos de trabalho melhor remunerados devido estes exigirem maior escolaridade e habilidades que muito raramente são alcançáveis por populações economicamente desfavorecidas ${ }^{(13,18,20)}$

Dessa forma, a pobreza é o horizonte mais provável na vida destas jovens, visto que é pouco provável Que elas retornem aos estudos, como aconteceu com parcela significativa de nossa amostra. Há literatura farta relacionando a gravidez na adolescência à perpetuação da pobreza, haja vista que o abandono dos estudos se renova na prole ${ }^{(20)}$, como é o caso da maioria das adolescentes de nosso estudo Que referiram outros casos de gravidez na adolescência de irmão ou irmã, cuja mãe também engravidou na adolescência e também tinha baixa escolaridade, o Que redunda o fato da jovem pertencer a família de baixa renda.

Outro aspecto negativo para a vida da jovem Que precocemente assume a vida conjugal Quando não tem renda própria é a dependência financeira de terceiros,

sexualmente ativas, ou mesmo grávidas, para Que não sejam socialmente estigmatizadas ou causem constrangimentos aos pais e à família, ou mesmo pode ser a saída à falta de oportunidades por meio de estudo e trabalho para o alcance dos objetivos da jovem, uma alternativa para assegurar seu futuro financeiro ${ }^{(16-17)}$. principalmente do companheiro. E considerando, Que ela tem grande probabilidade de abandonar os estudos, como já citado e comprovado, esta dependência financeira pode gerar um fator a mais, além da própria imaturidade de lidar com estas situações, Que é sofrer violência física e de outras naturezas tanto do parceiro 
Quanto de familiares ${ }^{(19)}$.

Quanto mais cedo a iniciação sexual da jovem, maiores as probabilidades de engravidar precocemente e de ter mais filhos e parceiros sexuais ${ }^{(20)}$. Apesar de Que para as jovens a virgindade não tenha tanta importância de ser mantida, tem sido mostrado Que maior atenção é dada à primeira relação sexual, com o planejamento desta "passagem", seja pela escolha do parceiro, seja mantendo o modelo feminino tradicional de opinião sobre sexo vinculando-o ao amor e afeto ${ }^{(21)}$.

Os dados sugerem Que a idade da primeira relação sexual tenha sido próxima à idade da menarca e mais distante da maioridade, pois mais de metade do grupo teve a primeira relação até os Quinze anos de idade. Importante chamar atenção para o fato de a idade de Quinze anos ser a única idade isolada de maior freeüência para Que estas jovens tenham tido sua primeira relação sexual com penetração, o denominado "debut sexual”. No Brasil, a idade de 15 anos tem um significado de passagem da fase de menina para a fase de mulher, Que é celebrado com uma festa especial, o baile de debutantes. Tradicionalmente, os pais são mais liberais com as garotas após elas completarem 15 anos e antes desta idade é comum Que pais prometam para a garota várias permissões, tal como poder sair sem a companhia deles à noite, Quando ela tiver 15 anos.

Tendo-se em conta Que não era objetivo deste estudo explorar profundamente este assunto, e diante de achado tão inusitado, sugere-se Que futuros estudos investiguem se em outros grupos sociais há esta freqüência aumentada do debut sexual nesta idade e se há relação entre o debut da idade de 15 anos e o debut sexual, bem como esclarecer os fatores Que levam a esta relação, caso esta se confirme.

A sexualidade na juventude tem sido considerada tanto pela ciência Quanto pelo poder público um ponto essencial para a redução de problemas de saúde pública relacionados ao tema. Assim, a educação sexual ganha vultuosidade teórica Que se divorcia da prática como pode ser constatado em achados na literatura ${ }^{(21-}$ 22). O tripé formado pela família, comunidade e escola Que deveriam teoricamente orientar a jovem nas suas escolhas sexuais e reprodutivas têm limitações severas para exercer sua função ${ }^{(23)}$. A família e a escola não têm se mostrado preparadas adequadamente para abordar o assunto e a comunidade, e Quando representada pelos serviços públicos de saúde também não ${ }^{(22)}$. A abordagem Quando é feita, na maioria das ocasiões, está centrada na biologia reprodutiva ou conselhos sem profundidade ${ }^{(21-22)}$. Outro ponto importante é Que a educação sexual deve ser provida antes da iniciação sexual da adolescente, devendo os envolvidos na tarefa estar convencidos de Que a educação sexual não incentiva a prática sexual e sim, torna-a consciente ${ }^{(23)}$. Como constatado em nosso estudo, a escola foi mais frequentemente apontada como instituição Que orientou a adolescente antes de sua iniciação sexual, euando comparada à família.

Assim, recomendamos Que as políticas públicas sejam elaboradas de forma a integrarem as instituições pilares da problemática social Que é a gravidez na adolescência. Incluir a família, capacitar os professores e disponibilizar serviços competentes para atenderem as demandas sexuais e reprodutivas dos jovens são fatores cruciais para Que se reduza a prevalência de gravidez precoce, tornando o jovem consciente de Que sexo tem conseqüência, seja a gravidez precoce e muitas vezes indesejada, sejam as doenças sexualmente transmissíveis ou alterações emocionais de variada monta ${ }^{(24-25)}$.

\section{CONCLUSÃO}

A reincidência de gravidez nos últimos dois anos de acordo com esse estudo ocorreu em uma parcela bem menor, o Que não deixa de ser preocupante, se levado em consideração Que o maior número de filhos pode reduzir mais ainda a possibilidade de retorno aos estudos desta jovem mãe, bem como aumentam as probabilidades de ter outra repetição rápida de gravidez. O aumento do risco de ter gestações repetidas também tem sido mostrado em decorrência delas terem um novo parceiro Que não o pai do bebê da gestação anterior, principalmente se ele ainda não tem filhos. Notou-se Que a primeira gestação de mais da metade de destas jovens repetiu o passado obstétrico de suas mães, o que pode ser um indício de Que a história obstétrica se repete por gerações.

Mesmo quando a gravidez ocorrer entre jovens estudantes, Que a escola por meio de todos Que a fazem - professores, alunos, funcionários e pais de alunos - seja inclusiva para estas jovens, pois assim, elas se sentirão bem-vindas e amparadas . Para isso é fundamental que os pais também sejam receptivos e a acolham, sem se importar com a repercussão Que a gravidez possa ter em suas relações sociais, pois somente dessa maneira, a jovem identificará Que a gravidez pode até dificultar sua vida e lhe trazer responsabilidades para as Quais ela não imaginava poder assumir, mas Que a tarefa será mais suave se ela puder contar com o respeito e o apoio de todos envolvidos nesse contexto.

Espera-se que a adolescente Que já tem um filho se sinta acolhida pela família, escola e profissionais de saúde, para Que alcance sucesso profissional almejado. Nesse sentido, a enfermagem, por excelência, contempla ações educativas de cunho social, tendo respaldo para gerenciar e desenvolver políticas educativas específicas para a clientela adolescente, seja em instituições hospitalares, escolares, bem como no âmbito coletivo utilizando a Estratégia de Saúde da Família.

\section{REFERÊNCIAS}

1. World Health Organization. Child and Adolescent Health Development. Geneva: WHO; 200 I . Disponível em: < http:// www.who.int/child-adolescent-health/OVERVIEW/AHD/adhover.htm > . Acesso em: abril, 2008.

2. Galleta MIC, Zugaib M. Pré-natal no PSF. 2001. Disponível em http://www.pré-natalnopsf.com.br. Acesso em 2007.

3. Instituto Brasileiro de Geografia e Estatística. Disponível em: http://www.ibge.gov.br/estatísticas de saúde e assistência médico sanitária. 2002. Acesso: 02/1 1/07.

4. Conselho Nacional dos Direitos da Criança e Adolescente. Estatuto da Criança e do Adolescente. Brasília: CONANDA; 2003.

5. Sekef G. Outro bebê a caminho. Veja 2001 ; 1706.

6. Hercowitz A. Gravidez na adolescência. Rev Pediatria Moderna 2002; 38(8): 74-5. 
7. Coates V, Sant'anna MIC. Gravidez na adolescência. In: Gejer D. Sexualidade e Saúde Reprodutiva na Adolescência. São Paulo: Atheneu; 2001. p. 71-84.

8. Belo MAV, Silva JLP. Conhecimento, atitude e prática sobre métodos anticoncepcionais entre adolescentes gestantes. Rev Saúde Pública 2004; 38(4): 479-87.

9. Fundação Municipal de Saúde. Informações em saúde. Súmula informativa. Teresina; 2006. [citado em 2008 Abril 10]. Disponível em: http://www.saude.teresina.pi.gov.br/ informacoes_em_saude/SUMULA_informativa_20052006_03.pdf

10. Ministério da Saúde (BR). Base de dados do SINASC. [citado em 2008 Abril 10]. Disponível em: http:// www.tabnet.datasus.gov.br/cgi/deftohtm.exe?sinasc/cnv/nvpi.def

11. Sabroza AR, Leal MC. Perfil sociodemográfico e psicossocial de puérperas adolescentes do município do Rio de Janeiro, Brasil - 1999 - 200 I. Cad Saúde Pública 2004; 20 (supl I): 112-20.

12. Chalem E, Mitsuhiro SS, Ferri CP, Barros MCM, Guinsburg R, Laranjeira R. Gravidez na adolescência: perfil sociodemográfico e comportamental de uma população da periferia de São Paulo, Brasil. Cad Saúde Pública 2007; 23(1): 177-86.

13. Gomes KRO. Who are the pregnant adolescents in the poorest state capital of Brazil? Public Health Nurs 2008; 25: 319-26.

14. World Health Organization. Marriage adolescents: no place of safety. 2006. [cited 2009 April 21]. Available from: http:// wholibdoc.who.int/publications/2006/9241593776_eng.pdf

15. World Bank. World Bank list of economies (July 2008). [cited 2009 April 21]. Available from: http://siteresources. worldbank.org/DATASTATISTICS/Resources/CLASS.XLS.

16. Mathur S, Greene M, Malhotra A. Too young to wed: the lives, rights and health of young married girls. International Center for Research on Women (ICRW). Washington; 2003. [cited 2009 April 21]. Available from: http://www.icrw.org/docs/ tooyoungtowed_1003.pdf

17. Gupta N. La formation des unions chez les adolescentes du Nordeste (Brésil). Cahiers Québécois Démographie 2000; 29(2): 287-306.

18. Almeida MCC, Aquino EML, Barros AP. School trajectory and teenage pregnancy in three Brazilian state capitals. Cad Saúde Pública 2006; 22(7): 1397-1409.

19. Haberland N, Chong E, Bracken H. Married adolescents: an overview. Geneva: WHO; 2003.

20. Altmann H. Educação sexual e primeira relação sexual: entre expectativas e prescrições. Est Feministas 2007; 15(2): 233 40.

21. Almeida ACCH, Centa ML. A família e a educação sexual dos filhos: implicações para a enfermagem. Acta Paul Enferm 2009; 22(I): 7I-6.

22. World Health Organization. Promoting and safeguarding the sexual and reproductive health of adolescents. [cited 2009 April 21]. Available from: http://www.who.int/reproductive-health/ publications/policybrief4.pdf

23. Persona L, Shimo AKK, Tarallo MC. Perfil de adolescentes com repetição da gravidez atendidas num ambulatório de pré natal. Rev Latino-am Enfermagem 2004; 12(5): 745-50.

24. Crittenden CP. The role of mental health factors, behavioral factors, and past experience in the prediction of rapid repeated pregnancy in adolescence. I Adolesc Health 2009; 44: 25-32.

25. Gomes KRO, Speizer IS, Oliveira DDC, Moura LNB, Gomes FM. Contraceptive method use by adolescents in brazilian state capital. J Pediatric Adolescent Gynecol 2008; 2 1: 2 13-19. 